BMJ Open

Diabetes

Research

\& Care

\title{
Inpatient diabetes management by specialized diabetes team versus primary service team in non-critical care units: impact on 30-day readmission rate and hospital cost
}

\author{
Vivek Bansal, ${ }^{1,2}$ Adham Mottalib, ${ }^{3}$ Taranveer K Pawar, ${ }^{4}$ \\ Noormuhammad Abbasakoor, ${ }^{5}$ Eunice Chuang, ${ }^{6}$ Abrar Chaudhry, ${ }^{7}$ \\ Mahmoud Sakr, ${ }^{3}$ Robert A Gabbay, ${ }^{3}$ Osama Hamdy ${ }^{3}$
}

\begin{abstract}
To cite: Bansal V, Mottalib A, Pawar TK, et al. Inpatient diabetes management by specialized diabetes team versus primary service team in non-critical care units: impact on 30-day readmission rate and hospital cost. BMJ Open Diab Res Care 2018;6:e000460. doi:10.1136/ bmjdrc-2017-000460
\end{abstract}

Received 10 August 2017 Revised 4 March 2018 Accepted 14 March 2018

Check for updates

${ }^{1}$ Department of Medicine, Beth Israel Deaconess Hospital-Needham, Needham, Massachusetts, USA

${ }^{2}$ Center for Advanced Weight Loss, Hunterdon Healthcare, Clinton, New Jersey, USA ${ }^{3} J o s l i n$ Diabetes Center, Harvard Medical School, Boston, Massachusetts, USA ${ }^{4}$ Department of Medicine, Lahey Clinic Medical Center, Boston, Massachusetts, USA ${ }^{5}$ Division of Endocrinology, Stanford University Medical Center, Stanford, California, USA

${ }^{6}$ Division of Endocrinology, University of California, San Francisco, California, USA ${ }^{7}$ Department of Medicine, Emory University, Atlanta, Georgia, USA

Correspondence to Dr Osama Hamdy; osama.hamdy@joslin.harvard. edu

\section{ABSTRACT}

Objective We compared the cost-effectiveness of two inpatient diabetes care models: one offered by a specialized diabetes team (SDT) versus a primary service team (PST).

Research design and methods We retrospectively evaluated 756 hospital admissions of patients with diabetes to non-critical care units over 6 months. Out of 392 patients who met the eligibility criteria, 262 were matched 1:1 based on the mean of the initial four blood glucose (BG) values after admission. Primary outcomes were 30-day readmission rate and frequency, hospital length of stay (LOS) and estimated hospital cost. Secondary outcomes included glycemic control and BG variability.

\section{Results Diabetes complexity and in-hospital}

complications were significantly higher among patients treated by SDT versus PST. Thirty-day readmission rate to medical services was lower by $30.5 \%$ in the SDT group versus the PST group $(\mathrm{P}<0.001)$, while 30 -day readmission rate to surgical services was $5 \%$ higher in the SDT group versus the PST group $(\mathrm{P}<0.05)$, but frequency of 30 -day readmissions was lower (1.1 vs 1.6 times, $P<0.05$ ). LOS in medical services was not different between the two groups, but it was significantly longer in surgical services in SDT $(P<0.05)$. However, LOS was significantly lower in patients who were seen by SDT during the first 24 hours of admission compared with those who were seen after that (4.7 vs 6.1 days, $P<0.001$ ). Compliance to follow-up was higher in the SDT group. These changes were translated into considerable cost saving.

Conclusions Inpatient diabetes management by an SDT significantly reduces 30 -day readmission rate to medical services, reduces inpatient diabetes cost, and improves transition of care and adherence to follow-up. SDT consultation during the first 24 hours of admission was associated with a significantly shorter hospital LOS.

\section{INTRODUCTION}

In 2014, about $9.3 \%$ (29.1 million) of the US population were presumed to have diabetes mellitus (DM). ${ }^{1}$ The American Diabetes

\section{Significance of this study}

What is already known about this subject?

- Hospital admissions for patients with diabetes are more likely to originate from emergency departments; they account for $20.3 \%$ of all US hospital readmissions and their hospital length of stay (LOS) is frequently longer. Having a higher hemoglobin A1c was shown to be associated with higher hospitalization cost.

What are the new findings?

- 30-Day readmission rate to non-critical medical units is reduced by around $30 \%$ when a specialized diabetes team (SDT) is involved in inpatient diabetes care in comparison with standard diabetes care by primary service team.

> SDT consultation within the first 24 hours of admission is associated with a significantly shorter LOS.

> Diabetes management by SDT reduces hospital cost and improves postdischarge follow-up adherence.

How might these results change the focus of research or clinical practice?

- This study suggests that more medical centers should consider using SDTs as part of their inpatient care for patients with diabetes. Benefits of SDT utilization warrant further investigation in prospective, long-term studies.

Association reported that the total cost of diabetes in 2012 was around $\$ 245$ billion (\$176billion in direct medical costs). ${ }^{2}$ About $43 \%$ ( $\$ 105.35$ billion) were attributed to hospital admissions. ${ }^{2}$ An estimated 43.1 million hospital days $(25.7 \%$ of the 168 million total hospital days) were incurred by patients with diabetes. Out of which, 
26.4 million days were attributed to diabetes as primary diagnosis. $^{2}$

Hospital admissions for patients with diabetes are more likely to originate from emergency departments (ED) and their hospital length of stay (LOS) is frequently longer. ${ }^{3}{ }^{4}$ Higher glycated hemoglobin A1c $\left(\mathrm{HbA}_{1 \mathrm{C}}\right)$, particularly $>10 \%$, was shown to be associated with higher hospitalization cost. ${ }^{5}$ Meanwhile, both hyperglycemia and hypoglycemia were shown to independently predict longer LOS. ${ }^{6}$ Improved glycemic control results in lower rates of in-hospital secondary complications. ${ }^{7}$ Reducing blood glucose (BG) variability was shown to be associated with reduced inpatient mortality, ${ }^{8}$ LOS, secondary complications ${ }^{9}$ and 30-day readmission rate. ${ }^{11}$ Prolonged exposure to hyperglycemia was also shown to be associated with higher risk of 30-day and 90-day readmission rates in patients with congestive heart failure (CHF). ${ }^{12}$

In most US hospitals, a primary service team (PST) is responsible for diabetes management in both medical and surgical non-critical care units and only few hospitals use specialized diabetes teams (SDT). Diabetes management by SDT usually includes diabetes evaluation and management by an endocrinologist or a diabetes nurse practitioner, general diabetes education by a dietitian and/or a certified diabetes educator followed by providing a diabetes discharge/transition plan and follow-up. This comprehensive diabetes care may be particularly valuable for patients with poorly controlled diabetes. However, it is unclear if comprehensive diabetes management by SDT is a better resource utilization when compared with diabetes management by PST. The main objective of this study is to compare the impact of the two different models of diabetes care on 30-day readmission rate and overall hospital cost in both medical and surgical non-critical care units.

\section{METHODS}

We conducted a comprehensive retrospective chart review of 756 consecutive patients with DM diagnosis admitted to the non-critical care units at a tertiary referral medical center in Boston, Massachusetts, USA, between July 2012 and January 2013. All non-pregnant patients aged 18 years and older with established diagnosis of DM were included in the study regardless of their primary admitting diagnosis (es). $\mathrm{HbA}_{1 \mathrm{C}}$ of $>6.5 \%$ or treatment with any antihyperglycemic medications was considered an additional confirmation of DM diagnosis. Newly diagnosed patients during hospital admission or patients with DM diagnosis of $<3$ months were excluded. We also excluded patients whose LOS is $<2$ days to ensure our ability to collect enough BG readings for this analysis. We presumed that within such short admission period, neither teams were to considerably impact study outcomes. We also excluded patients with long LOS of more than 10 days, as the average LOS in non-critical units in US hospitals is less than half of that duration (4.8 days). ${ }^{13}$ This strategy would mirror typical admissions and exclude outliers. All eligible patients who were readmitted within 30 days of their first admission or readmitted during the study period were only evaluated at their first admission.

During the 6-month study period, we identified 756 hospital admissions to non-critical care units with DM mentioned as primary, secondary or tertiary diagnosis on admission. Out of them, 392 patients were older than 18 years of age, non-pregnant and had an established DM diagnosis for $>3$ months. Out of these 392 patients, 262 were matched in 1:1 ratio (131 in each group) based on the mean value of their first four BG readings after admission. We used this criterion to ensure a common starting point for fair comparison between the two groups. We included in our analysis an equal number of patients managed in medical and surgical services for each group. The matching process was conducted by a biostatistician blinded to all study data except those values needed for matching as outlined.

Secondary medical complications specified for this study include new infections such as pneumonia or urinary tract infection, preoperative or postoperative infections, acute kidney injury (AKI), acute liver injury, myocardial infarction, CHF, acute cerebrovascular accident, gastrointestinal bleeding and transfer to intensive care unit (ICU). Diabetes complexity was defined as presence of type 1 diabetes diagnosis, $\mathrm{HbA}_{1 \mathrm{C}}>8.5 \%$ or presence of secondary medical complications during hospital admission.

\section{Models of care}

The PST group included patients managed by hospitalists, general internal/family medicine or general surgery physicians with or without involvement of house staff. The comparative group included patients managed by an SDT from Joslin Diabetes Center (JDC) who were consulted to manage DM. This team included an endocrinologist (with or without house staff or endocrinology fellow), a diabetes nurse practitioner, a certified diabetes nurse educator and discharge/transition coordinators. Comprehensive discharge pathway based on risk category was only offered in medical units, while routine discharge process was used in surgical units according to the hospital policy. This pathway indicates that patients with DM discharged on insulin are considered high-risk. A coordinated transition of DM care is synchronized between the medical center and JDC's outpatient clinic, where high-risk patients are scheduled to see their primary care physicians (PCPs), endocrinologists or diabetes nurse practitioners within 1-4 days of discharge and other patients are scheduled to see them within 2 weeks of discharge. General diabetes education and education assessments were provided for $30-60 \mathrm{~min}$ by a certified diabetes educator during hospital admission. Endocrinologists followed specified diabetes management protocols based on JDC guidelines for hospitalized patients. PST did not use any specific protocols in diabetes management. 


\section{Time of consultation}

To further evaluate the impact of SDT on DM management, we subdivided this group according to the time of initial consultation: (1) early SDT consultation: if requested SDT consults were initiated within the first 24 hours of admission; and (2) late SDT consultation: if requested consults were initiated after 24 hours of admission. This allowed us to properly evaluate the impact of SDT on hospital outcomes based on their time of involvement in DM management.

\section{Follow-up}

Patients' follow-up plans were obtained from discharge summaries. We were able to track if patients kept their follow-up appointments by reviewing providers' notes in the medical center's electronic health records (EHRs). However, we were only able to review this information for patients whose PCPs or endocrinologists are within the medical center's network of hospitals and clinics. Around $18 \%$ were outside this network. Due to this limitation, accurate assessment of follow-up adherence was deficient for some patients.

\section{Data collection}

During this study period, all capillary BG values during admission were captured to evaluate glycemic control, BG variability and episodes of documented hypoglycemia or hyperglycemia. For the SDT group, BG values were subdivided to before and after consultations. We defined hypoglycemia as $\mathrm{BG}<70 \mathrm{mg} / \mathrm{dL}$ and hyperglycemia as BG $>180 \mathrm{mg} / \mathrm{dL}$. BG variability was expressed as the SD of mean BG. All other clinical variables such as in-hospital secondary complications, mortality, LOS, 30-day readmission and primary diagnosis of first readmission were collected from the hospital EHR and scanned paper charts.

\section{Study outcomes}

The three primary outcomes of this study are 30-day readmission rate and frequency, LOS, and estimated hospital admission cost. Secondary outcomes include glycemic control, BG variability and incidence of hypoglycemia and hyperglycemia.

\section{Statistical methods}

Demographic and baseline characteristics were evaluated using descriptive statistics. All continuous variables were presented as means and SDs, and non-normally distributed variables were presented as medians and IQRs. All nominal/categorical variables were presented as frequencies and percentages. For comparisons between the two study groups, independent samples t-test was used for continuous variables. $\chi^{2}$ or Fisher's exact tests were used to compare categorical variables. Multivariate linear regression analysis was used to find predictors of the following numerical end-points: LOS and the total number of 30-day readmissions. Multivariate logistic regression analysis was used to define predictors of the following binary end-points: occurrence of
Table 1 Baseline characteristics of patients managed by the primary service team (PST) and by the specialized diabetes team (SDT)

\begin{tabular}{|c|c|c|c|}
\hline & PST & SDT & $P$ values \\
\hline$n$ & 131 & 131 & \\
\hline \multicolumn{4}{|l|}{ Sex (\%) } \\
\hline Male & 55 & 58 & NS \\
\hline Female & 45 & 42 & NS \\
\hline Age (years) & $69.1 \pm 11.1$ & $59.1 \pm 15.0$ & $<0.001$ \\
\hline $\begin{array}{l}\text { Admitted to medicine } \\
\text { service (\%) }\end{array}$ & 55 & 55 & NS \\
\hline $\begin{array}{l}\text { Admitted to surgery } \\
\text { service (\%) }\end{array}$ & 45 & 45 & NS \\
\hline $\begin{array}{l}\text { Average of the first four } \\
\text { blood glucose values } \\
\text { after admission (mg/dL) }\end{array}$ & $202.8 \pm 52.4$ & $202.6 \pm 60.5$ & NS \\
\hline $\begin{array}{l}\text { Most recent } \\
\text { hemoglobin A1c (\%) }\end{array}$ & $7.4 \pm 1.3$ & $8.7 \pm 2.1$ & $<0.001$ \\
\hline $\begin{array}{l}\text { Patients with type } 1 \\
\text { diabetes (\%) }\end{array}$ & 4.6 & 34.1 & $<0.001$ \\
\hline $\begin{array}{l}\text { Patients on oral } \\
\text { hypoglycemic } \\
\text { agents (\%) }\end{array}$ & 21.4 & 9.9 & 0.011 \\
\hline $\begin{array}{l}\text { Patients on basal } \\
\text { insulin (\%) }\end{array}$ & 45.8 & 58.0 & 0.048 \\
\hline $\begin{array}{l}\text { Patients on insulin } \\
\text { pump (\%) }\end{array}$ & 0 & 7.6 & $<0.001$ \\
\hline $\begin{array}{l}\text { Patients on insulin } \\
\text { drip (\%) }\end{array}$ & 0 & 3.0 & 0.004 \\
\hline $\begin{array}{l}\text { Patients with any } \\
\text { infection (\%) }\end{array}$ & 6.8 & 8.4 & NS \\
\hline $\begin{array}{l}\text { Patients with inpatient } \\
\text { complications (\%) }\end{array}$ & 20.6 & 60.6 & $<0.001$ \\
\hline
\end{tabular}

any complications during inpatient admission, provided follow-up referrals at time of discharge and readmitted within 30 days of discharge. For all methods mentioned above, $95 \%$ CIs were used and $\mathrm{P}$ values $<0.05$ were considered statistically significant. Stata V.12 software was used for data analysis.

\section{RESULTS}

There were no differences in gender between the two study groups, but patients in the PST group were significantly older $(\mathrm{P}<0.001) \quad$ (table 1$)$. Degree of diabetes complexity was significantly higher among patients treated by SDT versus PST $\left(\mathrm{HbA}_{1 \mathrm{C}} 8.7 \pm 2.1 \%\right.$ vs $7.4 \pm 1.3 \%$, respectively, $\mathrm{P}<0.001$ for both). In-hospital secondary complications were significantly higher among patients treated by SDT versus PST ( $60.6 \%$ vs $20.6 \%$, respectively, $\mathrm{P}<0.001)$. These complications include new infections, AKI, acute liver injury, myocardial infarction, CHF, acute cerebrovascular accident, gastrointestinal bleeding and transfer to ICU. Additionally, the SDT group had eight 
Table 2 Metabolic control during hospitalization and discharge disposition in patients managed by the primary service team (PST) and by the specialized diabetes team (SDT)

\begin{tabular}{|c|c|c|c|}
\hline & PST & SDT & $P$ values \\
\hline $\begin{array}{l}\text { Mean number of BG } \\
\text { monitoring during } \\
\text { hospitalization }\end{array}$ & $17.8 \pm 8.1$ & $23.4 \pm 10.9$ & $<0.001$ \\
\hline $\begin{array}{l}\text { Frequency of BG } \\
\text { monitoring per day }\end{array}$ & $3.8 \pm 1.0$ & $4.5 \pm 1.3$ & $<0.001$ \\
\hline $\begin{array}{l}\text { Mean of all BG values } \\
(\mathrm{mg} / \mathrm{dL})\end{array}$ & $193.3 \pm 43.0$ & $200.9 \pm 42.9$ & NS \\
\hline $\begin{array}{l}\text { Mean of the lowest } \\
\text { BG value }(\mathrm{mg} / \mathrm{dL})\end{array}$ & $110.5 \pm 33.9$ & $88.4 \pm 33.3$ & $<0.001$ \\
\hline $\begin{array}{l}\text { Frequency of } \\
\text { hypoglycemia } \\
\text { (BG }<70 \mathrm{mg} / \mathrm{dL}) \\
\text { per patient during } \\
\text { hospitalization }(\%)\end{array}$ & $0.2 \pm 0.8$ & $0.7 \pm 1.4$ & 0.01 \\
\hline $\begin{array}{l}\text { Mean of the highest } \\
\text { BG values }(\mathrm{mg} / \mathrm{dL})\end{array}$ & $300.2 \pm 84.9$ & $347.8 \pm 92.6$ & $<0.001$ \\
\hline $\begin{array}{l}\text { Frequency of } \\
\text { hyperglycemia } \\
\text { (BG >180 mg/dL) } \\
\text { per patient during } \\
\text { hospitalization (\%) }\end{array}$ & $8.5 \pm 6.1$ & $13.2 \pm 8.9$ & $<0.001$ \\
\hline $\begin{array}{l}\text { BG variability* } \\
(\mathrm{mg} / \mathrm{dL})\end{array}$ & 43.0 & 42.9 & NS \\
\hline $\begin{array}{l}\text { Discharge to } \\
\text { home (\%) }\end{array}$ & 41.2 & 38.2 & NS \\
\hline $\begin{array}{l}\text { Discharge to home } \\
\text { with care (VNA) (\%) }\end{array}$ & 36.6 & 42.0 & NS \\
\hline $\begin{array}{l}\text { Discharge to skilled } \\
\text { nursing facility (\%) }\end{array}$ & 11.5 & 0.76 & $<0.001$ \\
\hline $\begin{array}{l}\text { Discharge to } \\
\text { rehabilitation/district } \\
\text { hospital (\%) }\end{array}$ & 3.8 & 17.6 & $<0.001$ \\
\hline $\begin{array}{l}\text { Dead/expired during } \\
\text { hospitalization (\%) }\end{array}$ & 3.8 & 0.0 & 0.061 \\
\hline
\end{tabular}

${ }^{*} \mathrm{BG}$ variability is the difference between the highest and lowest readings during hospitalization.

BG, blood glucose; VNA, visiting nurses' service.

times the number of patients with type 1 diabetes $(34.1 \%$ vs $4.6 \%$, respectively, $\mathrm{P}<0.001)$. All patients on insulin pump in this cohort were managed by SDT as dictated by the medical center's internal policy. Baseline demographic data are outlined in table 1.

Rates of hyperglycemia and hypoglycemia were significantly higher in the SDT group $(\mathrm{P}<0.001)$ (table 2$)$. Diabetes consultation with SDT was initiated within the first 24 hours of admission in more than half of patients evaluated in this cohort. Surgical services were slightly more inclined to consider late consultations to SDT than medical services. There was significant improvement in average BG values pre-SDT versus post-SDT consults
(215 \pm 58 vs $192 \pm 44 \mathrm{mg} / \mathrm{dL}, \mathrm{P}<0.001)$. However, there was no difference in average $B G$ values after early versus after late consults in the SDT group. We also did not find any difference in average BG values between PST and SDT for either early or late consults. Patients with type 2DM followed by SDT had significantly lower BG variability (72 vs $87 \mathrm{mg} / \mathrm{dL}, \mathrm{P}<0.01$ ) than patients with type $1 \mathrm{DM}$ followed by the same team.

There was no difference in discharge disposition to home or home care with visiting nurse services between the two groups. However, PST had significantly higher rate of discharge to rehabilitation and skilled nursing facilities $(\mathrm{P}<0.001$ for both) (table 2$)$. More patients in the SDT group were referred to their PCPs and endocrinologists $(\mathrm{P}<0.001$ for both $)$. For patients with in-network trackable hospital follow-up visits, we found that patients seen by SDT were more likely to keep their appointments with their PCPs or endocrinologists after discharge ( $\mathrm{P}<0.001$ for both) (table 3$)$.

The 30-day readmission rate to medical services in the SDT group was $30.5 \%$ lower than the PST group (22.5 vs $32.4 \%$, respectively, $\mathrm{P}<0.001$ ), with non-significant difference in frequency of admission during 30 days (1.1 vs 1.2 times, respectively, $\mathrm{P}>0.05$ ). The 30 -day readmission rate to surgical services in the SDT group was higher than the PST group (26.7\% vs $21.7 \%$, respectively, $\mathrm{P}>0.05$ ), but the frequency of 30-day readmission was significantly lower ( 1.1 vs 1.6 times, $\mathrm{P}=0.015$ ) (table 4 ). The most common reasons for readmission are CHF, pneumonia and acute cardiovascular events. LOS was not different between the two groups (table 4). Patients who received SDT consultations within 24 hours of admission had a significantly lower LOS compared with patients who received SDT consultations after 24 hours ( 4.7 vs 6.1 days, $\mathrm{P}<0.001$ ) (figure 1 ).

Considering hospital cost, historical hospital data showed that 30-day readmission rates for all patients to non-critical medical and surgical services irrespective of diabetes diagnosis were $16.6 \%$ and $13.9 \%$, respectively, in 2011, and $15.1 \%$ and $9.7 \%$, respectively, in 2012. Based on a constructed cost model for a total of 6695 admissions with diabetes in 2011 and the 5567 admissions in 2012, we estimate that the full utilization of SDT for all patients with DM was to prevent 500 readmissions to medical services in 2011 and 416 readmissions in 2012 while increasing readmissions to surgical services by 36 in 2011 and 44 in 2012. Since the national average of hospital cost for patients with diabetes was $\$ 7830$ per admission in $2010,{ }^{14}$ we estimate that full utilization of SDT for all patients with DM in non-critical care units of the medical center would save $\$ 2.9$ million in hospital cost in 2011 and $\$ 3.5$ million in 2012 (table 5). ${ }^{14}$

\section{DISCUSSION}

Healthcare spending is progressively increasing and accounts for one-fifth of the US economy. ${ }^{15}$ In 2012, the Centers for Medicare and Medicaid Services started 
Table 3 Transition of care in patients managed by primary service team (PST) and by specialized diabetes team (SDT)

\begin{tabular}{lrrl}
\hline & PST & SDT & P values \\
\hline Transition of care (\% of total) & & & \\
All patients & & & \\
\hline PCP/surgery & 69.5 & 87.8 & $<0.001$ \\
\hline Endocrinologist & 2.3 & 32.8 & $<0.001$ \\
\hline Other specialists & 62.6 & 36.6 & $<0.001$ \\
\hline Medicine & & & \\
\hline PCP/surgery & 57.8 & 84.5 & $<0.001$ \\
\hline Endocrinologist & 1.4 & 46.5 & $<0.001$ \\
\hline Other specialists & 85.9 & 41.8 & $<0.001$ \\
\hline Surgery & & & \\
\hline PCP/surgery & 83.3 & 91.7 & NS \\
\hline Endocrinologist & 3.3 & 16.7 & 0.015 \\
\hline Other specialists & 37.7 & 28.8 & NS \\
\hline Compliance with transition & & & \\
(\% of total) & & & \\
\hline All patients & & & \\
\hline PCP/surgery & 46.6 & 67.2 & $<0.001$ \\
\hline Endocrinologist & 2.3 & 22.9 & $<0.001$ \\
\hline Other specialists & 41.2 & 29.7 & $\mathrm{NS}$ \\
\hline Medicine & & & \\
\hline PCP/surgery & 22.5 & 60.6 & $<0.001$ \\
\hline Endocrinologist & 1.4 & 32.4 & $<0.001$ \\
\hline Other specialists & 50.7 & 35.2 & $\mathrm{NS}$ \\
\hline Surgery follow & & & \\
\hline PCP/surgery & 75 & 75 & $\mathrm{NS}$ \\
\hline Endocrinologist & 3.3 & 11.7 & $\mathrm{NS}$ \\
\hline Other specialists & 30 & 23.3 & $\mathrm{NS}$ \\
\hline Ots & & & \\
\hline & & & \\
\hline & & & \\
\hline
\end{tabular}

Other specialists include physician specialties other than endocrinology not managing diabetes.

PCP, primary care physician.

to apply hospital penalties by reducing payments to hospitals for readmissions in an attempt to reduce national healthcare expenditure on diseases like heart failure, pneumonia and acute myocardial infarction. ${ }^{16}$ Uncontrolled hyperglycemia was shown to worsen outcomes and increase readmission rates in some of these comorbid conditions. ${ }^{12}$

SDT utilization was previously evaluated in several studies, which reported different outcomes. ${ }^{17-19}$ Results from one pilot study showed that SDT utilization resulted in reducing patients' 30 -day hospital readmission rate. ${ }^{17}$ However, another pilot study showed that patients managed by an SDT had reduced visits to ED, but with no change in readmission rates owing to patients' disease complexity as evident by their longer LOS. ${ }^{18}$ On the other hand, a recent study showed that SDT utilization for preoperative diabetes management improved glycemic control and reduced LOS. ${ }^{19}$
Table 4 30-Day readmission rate and length of stay in patients managed by primary service team (PST) and by specialized diabetes team (SDT)

\begin{tabular}{|c|c|c|c|}
\hline & PST & SDT & $P$ values \\
\hline Medicine (n) & 71 & 71 & \\
\hline $\begin{array}{l}\text { Total number of } \\
\text { readmissions in } 30 \text { days }\end{array}$ & 23 & 16 & $<0.05$ \\
\hline $\begin{array}{l}\text { Frequency of readmission } \\
\text { per patient }(\%)\end{array}$ & $1.2 \pm 0.4$ & $1.1 \pm 0.4$ & NS \\
\hline $\begin{array}{l}30-\text { Day readmission } \\
\text { rate }(\%)\end{array}$ & 32.4 & 22.5 & $<0.05$ \\
\hline Length of stay (days) & $4.8 \pm 1.9$ & $5.1 \pm 2.4$ & NS \\
\hline Surgery (n) & 60 & 60 & \\
\hline $\begin{array}{l}\text { Total number of } \\
\text { readmissions in } 30 \text { days }\end{array}$ & 13 & 16 & NS \\
\hline $\begin{array}{l}\text { Frequency of readmission } \\
\text { per patient }\end{array}$ & $1.6 \pm 0.7$ & $1.1 \pm 0.3$ & $<0.05$ \\
\hline $\begin{array}{l}\text { 30-Day readmission } \\
\text { rate }(\%)\end{array}$ & 21.7 & 26.7 & NS \\
\hline Length of stay (days) & $4.8 \pm 1.9$ & $5.6 \pm 2.2$ & $<0.05$ \\
\hline
\end{tabular}

Observed reduction in readmission rate among patients managed by SDT in this study, particularly in medical units, could be multifactorial. This may include better glycemic control, inpatient diabetes education (IDE), comprehensive discharge plan and appropriate transition of care. This notion is further supported by recent logistic regression models that elucidate formal IDE to be independently associated with lower frequency of all cause 30 -day readmission rates. ${ }^{20}$ One may argue that PST in this study cared for older patients who are more likely to have poorer outcome in general. However, the PST group represented a relatively healthier cohort with better baseline $\mathrm{HbA}_{1 \mathrm{C}}$ and a lower percentage of in-hospital secondary complications. On the other hand, patients with type $1 \mathrm{DM}$ were largely managed by the SDT group. Those patients tend to be younger, usually on insulin pumps, treated with complex insulin regimens and have higher BG variability. Therefore, higher frequencies of hypoglycemia and hyperglycemia in the SDT group were not surprising. It is known that readmission is traditionally higher among patients with higher $\mathrm{HbA}_{1 \mathrm{C}}$ on admission, patients with type $1 \mathrm{DM}$, and patients with multiple acute and chronic comorbid conditions. $^{21} 22$

In contrast to medical units, the readmission rate to surgical units was not improved in the SDT group. Diabetes education was provided to all patients in the SDT group in both medical and surgical units, so it is unlikely to contribute to any difference in outcomes between the two units. However, coordinated and comprehensive diabetes discharge plan with mandatory recommendations of PCPs or endocrinologists follow-up was only offered in medical units and might contribute to the observed difference in readmission between medical 


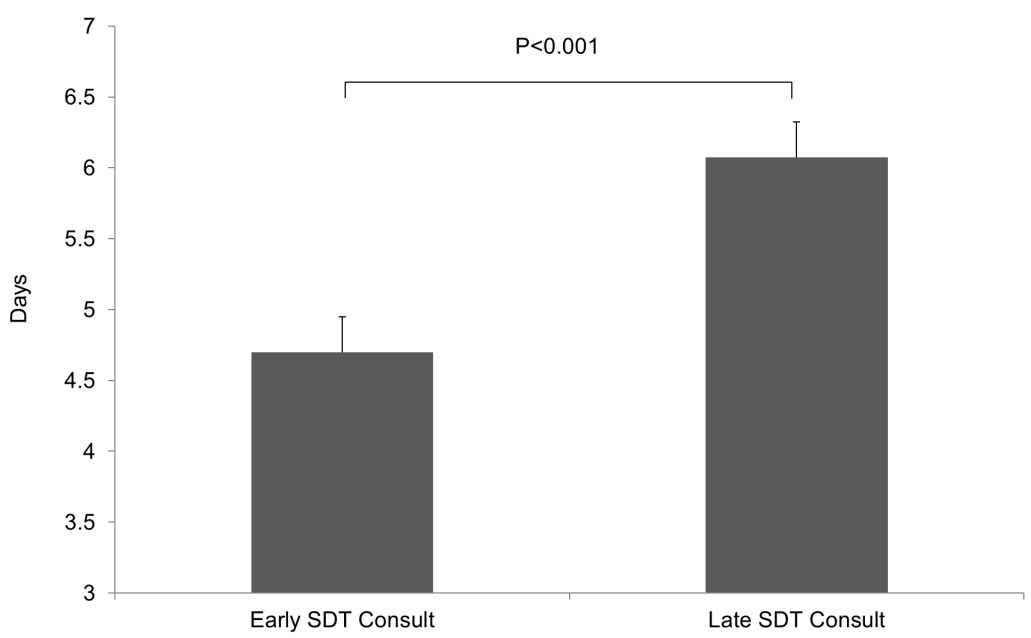

Figure 1 Hospital length of stay depending on the time of specialized diabetes team (SDT) consult after admission. Values are mean \pm SEM. Early: within 24 hours of admission; late: $>24$ hours of admission. $n=131,58 \%$ in the early SDT consult group and $42 \%$ in the late SDT consult group.

and surgical units. Involvement of SDT in DM management occurred after 24 hours of admission in over $50 \%$ of patients evaluated in this study. Trend of late consultations was higher in surgical units and might contribute to the observed differences in readmission rate between the two units. We rationally expect that earlier consultations to SDT during the hospital course may lead to better glycemic outcomes.

In this study, consulting SDT was appropriate for managing complex diabetes cases irrespective of the BG readings on admission. It was noticed that patients managed by the SDT were more likely to be referred to

Table 5 Postulated model of cost saving if specialized diabetes team (SDT) were to manage all patients with diabetes admitted in 2011 and 2012 in comparison with primary service team (PST) (model is calculated based on the actual admissions with diabetes diagnosis in 2011 and 2012)

\begin{tabular}{|c|c|c|c|}
\hline & PST & SDT & Difference \\
\hline \multicolumn{4}{|l|}{ Medicine } \\
\hline $\begin{array}{l}\text { Total number of admissions to the medical center with diabetes } \\
\text { diagnosis in } 2011\end{array}$ & 6695 & 6195 & -500 \\
\hline $\begin{array}{l}\text { Total cost of admissions adjusted for the average cost of } \$ 7830^{*} \\
\text { per admission (national average 2010) }\end{array}$ & $\$ 52421850$ & $\$ 48506850$ & $-\$ 3915000$ \\
\hline $\begin{array}{l}\text { Total number of admissions to the medical center with diabetes } \\
\text { diagnosis in } 2012\end{array}$ & 5567 & 5151 & -416 \\
\hline $\begin{array}{l}\text { Total cost of admissions adjusted for the average cost of } \$ 7830^{*} \\
\text { per admission (national average 2010) }\end{array}$ & $\$ 43589610$ & $\$ 40332330$ & $-\$ 3257280$ \\
\hline \multicolumn{4}{|l|}{ Surgery } \\
\hline $\begin{array}{l}\text { Total number of admissions to the medical center with diabetes } \\
\text { diagnosis in } 2011\end{array}$ & 1080 & 1124 & 44 \\
\hline $\begin{array}{l}\text { Total cost of admissions adjusted for the average cost of } \$ 7830^{*} \\
\text { per admission (national average 2010) }\end{array}$ & $\$ 8456400$ & $\$ 8800920$ & $\$ 344520$ \\
\hline $\begin{array}{l}\text { Total number of admissions to the medical center with diabetes } \\
\text { diagnosis in } 2012\end{array}$ & 880 & 916 & 36 \\
\hline $\begin{array}{l}\text { Total cost of admissions adjusted for the average cost of } \$ 7830^{*} \\
\text { per admission (national average 2010) }\end{array}$ & $\$ 6890400$ & $\$ 7172280$ & $\$ 281880$ \\
\hline Total cost 2011 & $\$ 60878250$ & $\$ 57307770$ & $-\$ 3570480$ \\
\hline Total cost 2012 & $\$ 50480010$ & $\$ 47504610$ & $-\$ 2975400$ \\
\hline
\end{tabular}

Cost is calculated based on the sum of fist admission and 30-day readmission for the same patients considering the 30-day readmission rate.

${ }^{*}$ Average national cost of admission to non-critical care unit for 2010. 
their PCPs and/or endocrinologists on discharge and were more likely to comply with their transition of care. This is an important step in preventing medication errors since medication reconciliation is required at various stages of patient care and may reduce frequency of unnecessary utilization of urgent or emergent care resources. In most if not all the hospitals across the USA, discharge instructions, follow-up plans and transition policies are handled by the PST, guided by hospital policy, patients' health insurance and availability of hospital services that handle transition of care. In this study, involvement of SDT increased the likelihood of appropriate transition of care and improved adherence to follow-up plans. Outpatient care following proper diabetes discharge plan by SDT in medical wards might prevent readmission since reduction in readmission was not seen in surgical wards for the same group, where there is no diabetes discharge plan or proper follow-up.

In this study, involvement of SDT in DM management had no impact on LOS, which is typically longer by 1 day for patients with DM compared with patients without DM. ${ }^{21}$ We found that hospital LOS was shorter in the PST group in comparison with the SDT group. This may be explained by the higher observed frequency of in-hospital secondary complications, larger number of patients with type $1 \mathrm{DM}$ and higher $\mathrm{HbA}_{1 \mathrm{c}}$ in the SDT group. Additionally, patients who were managed by SDT early in their hospital admissions had a significantly shorter LOS. It was long-debated which strategy has better positive impact on hospital cost: reducing 30-day readmission rate or reducing LOS. In the last few years, the Centers for Medicare and Medicaid Services, the largest insurer/payer in the USA, and many other insurance plans shifted toward reducing 30-day readmission rate rather than reducing LOS. ${ }^{23}$ It has been shown that single readmission usually costs more than an additional 1-2 days of hospital stay during first admission. ${ }^{24}$

This study suggests a considerable cost saving if SDT were to care for all patients admitted with DM diagnosis. Cost saving was slightly offset by increased readmissions to surgical units. Providing comprehensive discharge plans and transitions of care for surgical patients similar to those implemented for medical patients might contribute to additional cost saving.

This study has several limitations. First, it is a retrospective study conducted at a single, academic, tertiary care center and included a relatively small number of patients over a limited time period. The retrospective study design has the advantage of reporting accurate, real-life, hospital scenarios without any intention to change DM management behavior. However, this study design still has its own limitations, which include inability to properly match the comparative groups and inability to control timing of SDT consultation. Other clinical centers may have different protocols for diabetes care and different transition plans, so it is difficult to assume that these results are valid or reproducible in other hospitals. This study was not designed to evaluate newly diagnosed patients with DM during first admission, a patient population which may have different metabolic and hospital outcomes. It is assumed that this category of patients may benefit from basic diabetes education during admission and from structured diabetes discharge and transition plans. ${ }^{20}$ Although all readmissions were individually verified, it may be possible that readmissions to other hospitals occurred and were not reported in patients' EHR. For these reasons and to confirm our findings, we suggest a prospective randomized multicenter clinical study that neutralizes baseline variables for comparative groups, calculates actual costs and includes newly diagnosed patients and patients admitted to the ICU. Since patients with DM diagnosis account for $20.3 \%$ of all US hospital readmissions, ${ }^{14}$ this proposed study may be of great importance in our quest to reduce hospital cost of patients with DM.

In conclusion, this study suggests that using an SDT to manage diabetes in non-critical medical units may result in significant reduction in 30-day readmission rate in comparison with managing DM by a PST. Consultations of SDT should be initiated early after admission and preferably within the first 24 hours of admission in order to reduce LOS. Transition of DM care after discharge to PCPs and/or endocrinologists should be encouraged for all patients with DM discharged from either medical or surgical units to improve follow-up adherence and reduce 30-day readmission rate.

Acknowledgements The authors would like to acknowledge the support of the Office of Business Conduct and Quality at Beth Israel Deaconess Medical Center and the billing department of Joslin Diabetes Center for providing access to patients' data.

Contributors VB supervised study conduct and drafted its manuscript. AM contributed to statistical analysis, and reviewed and edited the manuscript. TKP did statistical analysis. NA, EC and AC collected patient data. MS shared in manuscript writing. RAG reviewed and edited the manuscript. $\mathrm{OH}$ supervised study conduct, shared in data analysis and edited the manuscript.

Funding The study was internally funded.

Competing interests $\mathrm{OH}$ receives research support from Abbott Nutrition, Novo Nordisk, Intarcia and the National Dairy Council; consults for Merck; and is on the advisory board of AstraZeneca. RAG consults for Onduo.

Patient consent Detail has been removed from this case description/these case descriptions to ensure anonymity. The editors and reviewers have seen the detailed information available and are satisfied that the information backs up the case the authors are making.

Ethics approval The study protocol was approved by the medical center's internal review board (Committee on Clinical Investigations, Beth Israel Deaconess Medical Center).

Provenance and peer review Not commissioned; externally peer reviewed. Data sharing statement No additional data are available.

Open Access This is an Open Access article distributed in accordance with the Creative Commons Attribution Non Commercial (CC BY-NC 4.0) license, which permits others to distribute, remix, adapt, build upon this work non-commercially, and license their derivative works on different terms, provided the original work is properly cited and the use is non-commercial. See: http://creativecommons.org/ licenses/by-nc/4.0/

(c) Article author(s) (or their employer(s) unless otherwise stated in the text of the article) 2018. All rights reserved. No commercial use is permitted unless otherwise expressly granted. 


\section{REFERENCES}

1. Centers for Disease Prevention and Control. National diabetes statistics report: estimates of diabetes and its burden in the United States. Atlanta, GA: US Department of Health and Human Services, 2014.

2. American Diabetes Association. Economic costs of diabetes in the U.S. in 2012. Diabetes Care 2013;36:1033-46.

3. Fraze T, Jiang HJ, Burgess J. Hospital Stays for Patients with Diabetes, 2008: Statistical Brief \#93. Healthcare Cost and Utilization Project (HCUP) Statistical Briefs. Rockville (MD: Agency for Healthcare Research and Quality (US), 2010.

4. Whitston M, Chung S, Henderson J, et al. What can be learned about the impact of diabetes on hospital admissions from routinely recorded data? Diabet Med 2012;29:1199-205.

5. Menzin J, Korn JR, Cohen J, et al. Relationship between glycemic control and diabetes-related hospital costs in patients with type 1 or type 2 diabetes mellitus. J Manag Care Pharm 2010;16:264-75.

6. Godar DA, Kumar DR, Schmelzer KM, et al. The impact of serum glucose on clinical outcomes in patients hospitalized with community-acquired pneumonia. WMJ 2011;110:14-20.

7. Umpierrez GE, Hellman R, Korytkowski MT, et al. Management of hyperglycemia in hospitalized patients in non-critical care setting: an endocrine society clinical practice guideline. J Clin Endocrinol Metab 2012;97:16-38.

8. Dungan KM, Binkley $P$, Nagaraja $\mathrm{HN}$, et al. The effect of glycaemic control and glycaemic variability on mortality in patients hospitalized with congestive heart failure. Diabetes Metab Res Rev 2011;27:85-93.

9. Monnier L, Colette C, Owens DR. Glycemic variability: the third component of the dysglycemia in diabetes. Is it important? How to measure it? J Diabetes Sci Technol 2008;2:1094-100.

10. Al-Dorzi HM, Tamim HM, Arabi YM. Glycaemic fluctuation predicts mortality in critically ill patients. Anaesth Intensive Care 2010;38:695-702

11. Ampudia-Blasco FJ, Ceriello A. [Importance of daily glycemic variability in achieving glycemic targets in type 2 diabetes: role of DPP-4 inhibitors]. Med Clin 2010;135(Suppl 2):33-9.

12. Dungan KM, Osei K, Nagaraja HN, et al. Relationship between glycemic control and readmission rates in patients hospitalized with congestive heart failure during implementation of hospital-wide initiatives. Endocr Pract 2010;16:945-51.

13. Kozak LJ, DeFrances CJ, Hall MJ. National hospital discharge survey: 2004 annual summary with detailed diagnosis and procedure data. Vital Health Stat 13 2006;162:1-209.

14. Healthcare Cost Utilization Project. Agency for healthcare research and quality. 2013. Sample weights: introduction to the HCUP Nationwide Emergency Department Sample (NEDS). 2009:13-14

15. Centers for Medicare \& Medicaid Services. National health expenditure data. 2012 http://www.cms.hhs.gov/ NationalhealthexpendData/downloads/tables.pdf

16. Axon RN, Williams MV. Hospital readmission as an accountability measure. JAMA 2011;305:504-5.

17. Koproski J, Pretto Z, Poretsky L. Effects of an intervention by a diabetes team in hospitalized patients with diabetes. Diabetes Care 1997;20:1553-5.

18. Ostling S, Wyckoff J, Ciarkowski SL, et al. The relationship between diabetes mellitus and 30-day readmission rates. Clin Diabetes Endocrinol 2017;3:3.

19. Garg R, Schuman B, Bader A, et al. Effect of Preoperative Diabetes Management on Glycemic Control and Clinical Outcomes after Elective Surgery. Ann Surg 2017:1.

20. Healy SJ, Black D, Harris C, et al. Inpatient diabetes education is associated with less frequent hospital readmission among patients with poor glycemic control. Diabetes Care 2013;36:2960-7.

21. Enomoto LM, Shrestha DP, Rosenthal MB, et al. Risk factors associated with 30-day readmission and length of stay in patients with type 2 diabetes. J Diabetes Complications 2017;31:122-7.

22. Zekry D, Loures Valle BH, Graf C, et al. Prospective comparison of 6 comorbidity indices as predictors of 1-year post-hospital discharge institutionalization, readmission, and mortality in elderly individuals. J Am Med Dir Assoc 2012;13:272-8.

23. Chen LM, Jha AK, Guterman S, et al. Hospital cost of care, quality of care, and readmission rates: penny wise and pound foolish? Arch Intern Med 2010;170:340-6.

24. Carey K. Measuring the hospital length of stay/readmission cost trade-off under a bundled payment mechanism. Health Econ 2015;24:790-802. 